\title{
VARIACIONES ESTACIONALES DE LAS ALGAS EPIFÍTICAS DE MACRÓFITOS EN ALGUNOS SISTEMAS LACUSTRES DE CATALUÑA

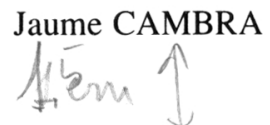

RESUMEN. Se han estudiado las variaciones estacionales de las poblaciones epifíticas en diversos macrófitos de Cataluña (N.E. de España) durante el año 1988. En total, se han identificado 204 táxones. La máxima diversificación de especies epifíticas se situa en primavera y al principio de verano, siendo las Cianofíceas, Bacilariofíceas y Clorofíceas los grupos más representados. En otoño, se observa un fuerte descenso en la riqueza florística de las poblaciones epifíticas.

Palabras clave. Algas epifíticas, poblaciones, macrófitos, variaciones estacionales.

ABSTRACT. The seasonal variations of epiphytic communities on several macrophytes were studied during 1988 in some lakes of Catalonia (N.E. of Spain). A total of 204 taxa were recorded. A rich algal flora was observed mainly in spring and early summer. Among them, the Cyanophyceae, Bacillariophyceae and Chlorophyceae were the most important groups. In autumn, a floristic richness strongly decreased.

Key words. Epiphytic algae, community, macrophyte, seasonal variation.

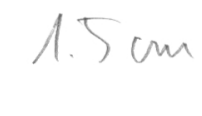

\section{INTRODUCCIÓN}

Como continuación de una serie de aportaciones al conocimiento de las algas epifíticas en España (Cambra, 1987, 1991 a,b,c), en este trabajo se aborda el estudio de los cambios estacionales de éstas poblaciones algales.

Las variaciones en la composición específica de estas poblaciones en las diferentes épocas del año han merecido la atención de numerosos ficólogos. Moss (1976) comparó la sucesión de las diatomeas epifíticas en lagunas enriquecidas con nutrientes. Klarer \& Hickman (1975) examinaron las relaciones entre la temperatura y la sucesión de algas epifíticas. Así mismo, numerosos estudios han aportado datos sobre el estudio de la sucesión en substratos vegetales, generalmente macrófitos (Cattaneo \& Kalff, 1979; Jones \& Mayer, 1983; Kairesalo, 1984) y en substratos artificiales (Brown \& Austin, 1973; Stockner \& Armstrong, 1971).

En el presente trabajo, se han estudiado en el período de un año las poblaciones epifíticas de 8 especies de macrófitos distribuídos en 7 sistemas lacustres de Cataluña. Se ha dedicado especial atención a la riqueza florística en las diferentes estaciones del año. Los datos referentes a la flora epifítica de cada macrófito se recogen en Cambra (1991b). 


\section{METODOLOGÍA}

El presente estudio se realizó en sistemas lacustres de Cataluña (Tabla I). Se recogieron tres ejemplares de cada macrófito, obteniéndose diversas submuestras de las algas epifíticas a partir de tallos y hojas. En cada recolección, el material se obtuvo desde el litoral y en las distintas épocas del año siempre desde el mismo punto. Las muestras eran fijadas inmediatamente en el campo con formaldehído al $4 \%$. Una descripción física y limnológica de estos sistemas se recoge en Cambra (1991a, b). Se realizaron dos campañas de recolección en cada estación del año. Para su estudio al microscopio óptico (OLYMPUS CH-2) se realizaron 10 preparaciones de la epidermis de cada muestra recolectada. Así mismo, de cada fragmento se separó una aliquota con la finalidad de limpiar de materia orgánica las bacilariofíceas en base el protocolo seguido por Tomàs (1979). Los frústulos se montaron en medio de montaje Naphrax.

1.- Altafulla (Tarragonès, 31TCF6354). Chara hispida, Ruppia maritima.

2.- Banyoles (Pla de l'Estany, 31TDG8005). Ceratophyllum demersum, Cladium mariscus, Myriophyllum spicatum,Typha angustifolia.

3.- Basturs (Pallars Jussà, 31TCG3667). Ceratophyllum demersum, Cladium mariscus, Myriophyllum spicatum.

4.- Cap de Creus (Alt Empordà, 31TEG18). Fontinalis hypnoides.

5.- Estanya (Baixa Ribagorça, 31TBG9556). Chara polyacantha, Cladium mariscus, Typha angistifolia.

6.- Sant Miquel (Pallars Jussà, 31TCG16). Chara hispida, Typha angustifolia .

7.- Tancada (Montsià, 31TCF0901). Ruppia maritima.

Tabla I. Localidades estudiadas.

\section{RESULTADOS}

El catálogo florístico que se presenta consta de 204 táxones, distribuídos en 31 cianofíceas, 8 euglenofíceas, 4 dinofíceas, 5 crisofíceas, 86 bacilariofíceas, 7 tribofíceas, 4 rodofíceas, 1 glaucofícea, 40 clorofíceas y 18 zignematofíceas. A continuación se relacionan los táxones identificados, con indicación del macrófito donde han sido observados (Cd:Ceratophyllum demersum; Ch: Chara hispida; $\mathrm{Cp}$ :Charapolyacantha; $\mathrm{Cm}$ : Cladium mariscus; Fh: Fontinalis hypnoides; Ms: Myriophyllum spicatum; Rm: Ruppia maritima; Ta: Typha angustifolia).

\section{CYANOPHYCEAE}

Anabaena aequalis Borge: Cd.

Anabaena ambigua Rao: Cd.

Aphanocapsa littoralis Hansg.: Rm.

Aphanothece stagnina (Spreng.) Braun: $\mathrm{Cm}$.

Calothrix clavata G.S. West: Rm.

Chroococcus minutus (Kütz.) Näg.: $\mathrm{Ch}, \mathrm{Cm}, \mathrm{Ms}$, Ta.

Chroococcus turgidus (Kütz.) Näg.: Ch, Cm, Ms.

Cylindrospermum minutissimum Collins: Ch, Ta.

Gloeocapsa decorticans (A. Braun) P. Kicht: Ta. 
Gloeocapsa granosa (Berkel.) Kütz.: Cm.

Gloeothece confluens Näg.: Cp.

Gloeothece vibrio N. Carter: Cp.

Gomphosphaeria aponina Kütz.: Cm.

Homeothrix juliana (Menegh.) Kirch.: $\mathrm{Cm}$.

Lyngbya epiphytica Hieron: Rm.

Lyngbya halophila Hansg.: Ta.

Lyngbya kützingii Schmidle: $\mathrm{Cd}, \mathrm{Ch}, \mathrm{Rm}$.

Merismopedia minima G. Beck: Ta.

Merismopedia punctata Meyen: Cd, Ch.

Nodularia spumigera Mertens: Ta.

Nostoc sphaericum Vaucher: Fh.

Oscillatoria limosa (Roth) C.A. Ag.: Ms.

Oscillatoria princeps Vaucher: Ch.

Oscillatoria splendida Grev.: $\mathrm{Cm}$.

Oscillatoria tenuis C.A. Ag.: Cp.

Phormidium ramosum Boye-Petersen: $\mathrm{Cp}$.

Rivularia beccariana (De Not.) Born. et Flah.: Fh.

Spirulina subtilissima Kütz.: Cp.

Tolypothrix distorta Kütz.: Cd, Fh, Ta.

Tolypothrix tenuis (Kütz.) J. Schmidt: Cm, Ms.

Xenococcus minimus Geitler: Cd.

\section{EUGLENOPHYCEAE}

Euglena proxima Dangeard: Ta.

Euglena sanguinea Ehr.: Ch.

Phacus caudatus Hübner: Ta.

Trachelomonas hispida (Pery) Stein: Ta.

Trachelomonas oblonga Lemm.: Ta.

Trachelomonas pusilla Playfair: $\mathrm{Ch}$.

Trachelomonas rugulosa Stein: $\mathrm{Ch}$.

Trachelomonas volvocina $\mathrm{Ehr} . \mathrm{Ch}$, Ta.

\section{DINOPHYCEAE}

Ceratium cornutum (Ehr.) Clap. et Lach.: Ms.

Gymnodinium neglectum (Schilling) Lind.: Ta.

Peridinium cinctum (O.F. Müller) Ehr.: Ch, Ta.

Peridinium umbonatum Stein: $\mathrm{Ch}$.

\section{CHRYSOPHYCEAE}

Chromulina truncata Conrad: Ch.

Chrysopyxis bipes Stein: Ms.

Chrysopyxis iwanoffii Lauter.: Ms.

Dinobryon sertularia Ehr.: Ta.

Sphaeridiothrix compressa Pascher \& Vlk: Cd.

\section{BACILLARIOPHYCEAE}

Achnanthes affinis Grun.: $\mathrm{Cm}$.

Achnanthes brevipes $v$. intermedia (Kütz.) Cleve: Cd.

Achnanthes brevipes $v$. parvula $\mathrm{Kütz}$.: Cd. 
Achnanthes coarctata (Bréb.) Grun.: Fh, Ta.

Achnanthes flexella (Kütz.) Brun: $\mathrm{Cm}$.

Achnanthes lanceolata Bréb.: Fh.

Achnanthes linearis (W. Smith) Grun.: Cm.

Achnanthes minutissima Kütz.: $\mathrm{Cd}, \mathrm{Ch}, \mathrm{Cm}, \mathrm{Ta}$.

Achnanthes plönensis Hustedt: Cm.

Amphipleura pellucida (Kütz.) Kütz.: Cd, Ta.

Amphora coffeaeformis (C.A. Ag.) Kütz.: Cp.

Amphora normanii Rabenh.: Cp.

Amphora pediculus (Kütz.) Grun.: $\mathrm{Cp}, \mathrm{Cm}$.

Amphora veneta Kütz.: $\mathrm{Cp}$.

Anomoeoneis vitrea (Grun.) Ross: $\mathrm{Cp}$.

Bacillaria paradoxa Gmelin: Cd.

Caloneis alpestris (Grun.) Cleve: $\mathrm{Cp}$.

Caloneis bacillum (Grun.) Cleve: Cp.

Caloneis latiuscula $\mathrm{Ehr} .: \mathrm{Cm}$.

Cocconeis diminuta Pant.: $\mathrm{Cm}$.

Cocconeis pediculus Ehr.: Cd, Rm.

Cocconeis placentula Ehr.: Cd, Cp, Fh, Rm, Ta.

Cyclotella kützingiana Thw.: Cd, Cp, Cm.

Cyclotella meneghiniana Kütz.: Cd, Cp.

Cyclotella ocellata Pant.: Cm.

Cymbella amphicephala Näg.: Cm.

Cymbella cesatii (Rabenh.) Grun.: $\mathrm{Cp}$.

Cymbella cistula (Ehr.) Kirchner: $\mathrm{Cm}$.

Cymbella cymbiformis v. nonpunctata Font.: Cp.

Cymbella helvetica Kütz.: $\mathrm{Cm}$.

Cymbella lanceolata (C.A. Ag.) C.A. Ag.: Cd, Ta.

Cymbella silesiaca Bleisch: $\mathrm{Cp}, \mathrm{Cm}$.

Cymbella subaequalis Grun.: $\mathrm{Cp}, \mathrm{Cm}$.

Diploneis ovalis (Hilse) Cleve: $\mathrm{Cp}, \mathrm{Cm}$, Ta.

Epithemia adnata (Kütz.) Bréb.: Cd, Cp, Fh, Ta.

Epithemia muelleri Fricke: $\mathrm{Cm}$.

Epithemia sorex Kütz.: Cd, Cp, Fh, Ta.

Epithemia turgida (Ehr.) Kütz.: Cd, Cp, Fh, Ta.

Eunotia arcus Ehr.: Cm.

Eunotia exigua (Bréb.) Rabenh.: $\mathrm{Cm}$.

Eunotia glacialis Meister: $\mathrm{Cm}$.

Eunotia parallela $\mathrm{Ehr} . \mathrm{Cm}$.

Eunotia pectinalis (Kütz.) Rabenh.: Cd, Fh.

Eunotia praerupta Ehr.: Cp.

Eunotia valida Hustedt: Cm.

Fragilaria brevistriata Grun.: $\mathrm{Cm}$.

Fragilaria construens v. venter (Ehr.) Grun.: Ch.

Fragilaria lapponica Grun.: Cd, $\mathrm{Cp}, \mathrm{Cm}$.

Gomphonema acuminatum Ehr.: Cd, Fh, Ta.

Gomphonema angustum C.A. Ag.: Cm.

Gomphonema clavatum Ehr.: Cp.

Gomphonema gracile Ehr.: Cp.

Gomphonema parvulum Kütz.: Cd, Ch.

Gomphonema truncatum Ehr.: Cd, Ch, Fh, Ta.

Hantzschia spectabilis (Ehr.) Hustedt: Cp.

Mastogloia elliptica v. dansei (Thw.) Cleve: Cp.

Mastogloia smithii v. lacustris Grun.: Cp.

Melosira juerguensii v. subangularis Grun.: Rm.

Melosira varians C.A. Ag.: Cd, Fh, Ta.

Meridion circulare (Grev.) C.A. Ag.: Cd, Fh, Ta. 
Navicula cincta (Ehr.) Ralfs: $\mathrm{Cm}$.

Navicula cryptocephala Kütz.: Cm, Ta.

Navicula halophila (Grun.) Cleve: $\mathrm{Cd}$.

Navicula radiosa Kütz.: Fh, Ta.

Navicula rhyncocephala Kütz.: $\mathrm{Cm}$.

Navicula stroemii Hustedt: $\mathrm{Cm}$.

Navicula subminuscula Manguin: $\mathrm{Cm}$.

Nitzschia frustulum Ehr.: Cp.

Nitzschia hungarica Grun.: Ta.

Nitzschia linearis (C.A. Ag.) W. Smith: Fh, Ta.

Nitzschia palea (Kütz.) W. Smith: Cp.

Nitzschia paleacea (Grun.) Grun.: Cp.

Nitzschia pusilla Grun.: Cp.

Nitzschia sigmoidea (Nitzsch) W. Smith: Cp.

Nitzschia vermicularis (Kütz.) Hantzsch: Cp.

Pinnularia gibba Ehr.: Cd, Ta.

Pinnularia maior (Kütz.) Rabenh.: Cp.

Pinnularia microstauron (Ehr.) Cleve: $\mathrm{Cp}$.

Pleurosigma elongatum W. Smith: Rm.

Rhopalodia gibba (Ehr.) O. Müller: Cd, Cp, Fh, Ta.

Rhopalodia musculus (Kütz.) O. Müller: Rm.

Rhopalodia rupestris (W. Smith) Krammer: Cp.

Synedra acus Kütz.: Cp, Fh, Ta.

Synedra capitata Ehr.: $\mathrm{Cp}, \mathrm{Cm}$.

Synedra tabulata (C.A. Ag.) Kütz.: Cd.

Synedra ulna (Nitzsch) Ehr.: Cd, Ch, Cp.

\section{TRIBOPHYCEAE}

Ophiocytium cochleare (Eichwald) A. Braun: Cd, Ta.

Tribonema affine (G. West) G. West: Ta.

Tribonema minus (Klebs) Hazen: Cd, Fh.

Tribonema spirotaenia Ettl: Ms.

Tribonema viride Pascher: Ta.

Tribonema vulgare Pascher: Rm, Ta.

Vaucheria sessilis (Vaucher) De Candolle: Ta.

\section{RHODOPHYCEAE}

Ceramium diaphanum (Lighfoot) Roth: Rm.

Chondria tenuissima (Gooden. \& Wood.) C.A. Ag.: Rm.

Compsopogon coeruleus (Balbis) Montagne: Cd, Ta.

Polysiphonia sp.: Rm.

\section{GLAUCOPHYCEAE}

Glaucocystis nostochinearum Itzigs.: Ch.

\section{CHLOROPHYCEAE}

Aphanochaete repens A. Braun: Cd, Fh, Ta.

Binuclearia tectorum (Kütz.) Beger: $\mathrm{Cd}, \mathrm{Ch}, \mathrm{Ms}, \mathrm{Ta}$.

Bulbochaete mirabilis Wittrock: Cd.

Chaetophora elegans (Roth) C.A. Ag.: Cd, Fh, Ta. 
Chaetosphaeridium pringsheimii Klebahn: Cd.

Characium ensiforme Hermann: Ta.

Cladophora fracta (Müller) Kütz.: Ch.

Coelastrum astroideum De Not.: Ms.

Coenocystis planctonica Kors.: Ta.

Coleochaete scutata Bréb.: Cd, Fh.

Crucigenia tetrapedia (Kirch.) W. \& G.S. West: Ms.

Draparnaldia mutabilis (Roth) Ceder.: Cd, Fh.

Epibolium dermaticola Printz: Fh.

Gloeocystis vesiculosa Näg.: $\mathrm{Cm}$, Ta.

Gloeoplax weberi Schmidle: Cd, Cm.

Golenkinia brevispina Kors.: Ms.

Gongrosira disciformis Fritsch: $\mathrm{Cm}$.

Microspora quadrata Hazen: Ms.

Microthamnion strictissimum Rabenh.: Ms.

Monoraphidium arcuatum (Kors.) Hindak: Rm.

Monoraphidium contortum (Thur.) Komarková-Leg.: Ms.

Monoraphidium pseudobraunii (Belcher \& Swale) Heynig: Ta.

Nephrocytium agardhianum Näg.: Ch, Ta.

Nephrocytium lunatum W. West: Ms.

Oedogonium crispum (Hassall) Wittrock: Cd, Ms.

Oedogonium khannae Skuja: Ch.

Palmella mucosa Kütz.: Ta.

Palmodyction lobatum Kors.: Cp.

Pandorina morum (O.F. Müller) Bory: Ch.

Pediastrum boryanum (Turpin) Menegh.: Cm, Ms.

Pediastrum integrum Näg.: Cm, Ms.

Protoderma viride Kütz.: Cd, Ms.

Scenedesmus acutus Meyen: Ta.

Scenedesmus ecornis (Ehr.) Chodat: Cd, Ms.

Scenedesmus obliquus (Turpin) Kütz.: Ch, Ta.

Scenedesmus ovalternus Chodat: Ms, Ta.

Scenedesmus ovalternus v. graevenitzii (Bernard) Chodat: Rm.

Scenedesmus sempervirens Chodat: Ms.

Sphaerocystis schroeteri Chodat: Ms.

Stigeoclonium tenue (C.A. Ag.) Kütz.: Cd.

\section{ZYGNEMATOPHYCEAE}

Closterium cynthia De Not.: Ch.

Cosmarium botrytis Menegh.: Ch, Ta.

Cosmarium brebissonii Menegh.: $\mathrm{Cm}$, Ms.

Cosmarium laeve Rabenh.: Cd, Ch, Cm, Ms.

Cosmarium rectangulare Grun.: Ms.

Cosmarium succissum W. West: Ta.

Cosmarium undulatum Corda: $\mathrm{Ch}$.

Cosmocladium pusillum Hilse: Ch.

Euastrum erosum Lundell: $\mathrm{Cp}$.

Hyalotheca dissilens (J.E. Smith) Bréb.: Cd, Fh.

Sirogonium sticticum (Engl. Bot.) Kütz.: Ta.

Spirogyra parvula (Transeau) Czurda: Ch, Ta.

Staurastrum gracile Ralfs: Ch.

Staurastrum punctulatum Bréb.: Ta.

Staurastrum striolatum (Näg.) Archer: Ch, Ta.

Staurodesmus dejectus (Bréb.) Teiling: Ms.

Zygnema kazachstanicum (Rund) Kadl.: Ch.

Zygnema subcylindricum Krieger: $\mathrm{Ch}$, Ta. 
Por lo que se refiere a las variaciones estacionales de las algas epifíticas, en general, se observa que en los meses correspondientes a la primavera existe una elevada diversificación de especies epifíticas, excepto en Chara hispida y Myriophyllum spicatum, especies que presentan el máximo número de algas epifíticas situado en verano.

De los grupos de algas estudiados, las cianofíceas, bacilariofíceas y clorofíceas son los más representados (Cambra, 1991b). Por lo que se refiere a las cianofíceas, se aprecia que pueden formar poblaciones más o menos estables en el tiempo (p.ej. Ceratophyllum demersum, Typha angustifolia) o, en cambio, presentar una mayor diversificación de especies en primavera (p.ej. Ruppia maritima) o en verano (p.ej. Chara hispida, Cladium mariscus, Myriophyllum spicatum). En este último caso se observaron numerosas cianofíceas (p.ej. Chroococcus minutus, C. turgidus, Merismopedia punctata), hecho que podría estar relacionado con el aumento del grado de eutrofia que se observa en esta época del año (Cambra, 1991a,b,c).

Las bacilariofíceas son el grupo mejor representado por lo que hace al número de especies. Éstas son abundantes en todos los macrófitos estudiades, aunque muchas de ellas provienen del plancton o del herpon, las formas fijas también presentan un número respetable. Géneros como Cocconeis y Achnanthes colonizan en aproximadamente dos semanas cualquier substrato submergido. En general, se observa un mayor número de especies en primavera (p.ej. Ceratophyllum demersum, Chara polyacantha, Cladium mariscus, Fontinalis hypnoides) y, ocasionalmente, en verano (p.ej. Chara hispida, Myriophyllum spicatum).

Las clorofíceas se presentan también en casi todas las épocas del año, destacando por su abundancia Aphanochaete repens, Gloeoplax weberi y Protoderma viride. En primavera el número de especies de clorofíceas es elevado (p.ej. Ceratophyllum demersum, Ruppia maritima, Typha angustifolia), aunque en algunos casos, en verano también se observa una diversificación importante (p.ej. Chara hispida, Cladium mariscus, Myriophyllum spicatum). Un caso particular se observa en Fontinalis hypnoides, planta que presenta un número máximo de clorofíceas en invierno. Por otra parte, Chara polyacantha destaca por la práctica inexistencia de clorofíceas en su superfície.

A continuación se comenta, para cada macrófito estudiado, la dinámica estacional de las poblaciones de epífitos que se desarrollan en su superfície.

\section{Ceratophyllum demersum L. (Fig. 1; Loc.: 2, 3)}

Este hidrófito aparece a finales de invierno y en otoño las plantas se degradan por completo. Los ejemplares estudiados s han observado en lagos de tipo cárstico, en aguas con una alcalinidad de $1.36-4.81$ meq..$^{-1}$, cuya temperatura osciló entre $14.7-27.8^{\circ} \mathrm{C}$, el pH entre 6.5-7.8 y el oxígeno disuelto entre 7.1-9.6 mg. $1^{-1}$. La conductividad fue variable, situada entre los 478-4200 $\mu \mathrm{S} . \mathrm{cm}^{-1}$, hecho que se puede atribuir al elevado contenido en sales de los medios estudiados, por un lado bicarbonatos y por el otro cloruros $\left(25-1850 \mathrm{mg}^{-\mathrm{l}^{-1}}\right)$ y sulfatos $(40-575$ mg. $\left.1^{-1}\right)$.

A finales de invierno, su superfície se encuentra recubierta por diversos clorófitos como Binuclearia tectorum, Coleochaete scutata, Gloeoplax weberi, Protoderma viride y, sobre todo, por Aphanochaete repens, así como por numerosas diatomeas como Epithemia turgida, Melosira varians y Rhopalodia gibba.

Durante la primavera se alcanza la mayor riqueza en especies epifíticas. A finales de ésta estación y, principalmente, durante el verano, observamos poblaciones importantes de Cocconeis placentula, especie abundante en esta época del año. Acompañando a Cocconeis hay otras diatomeas como Achnanthes brevipes, Cyclotella kützingiana, Synedra sp. pl. y la cianofícea Lyngbya epiphytica. 


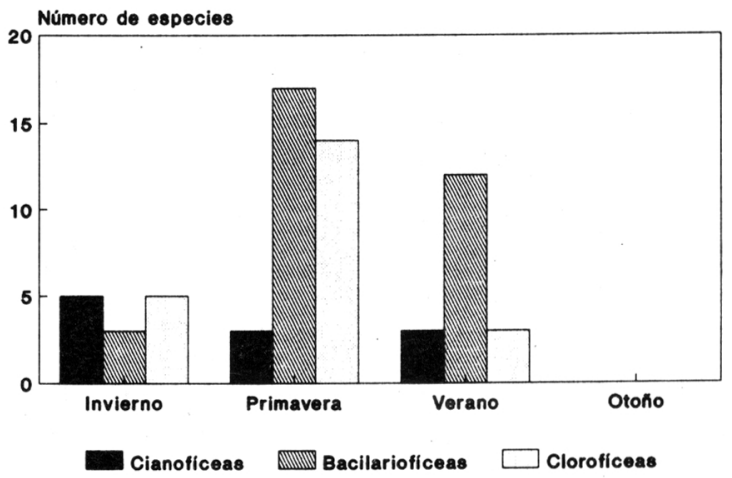

Fig. 1. Variación estacional del número de especies de cianofíceas, bacilariofíceas y clorofíceas epifíticas de Ceratophyllum demersum.

Chara hispida L. (Fig. 2; Loc.: 1, 6)

Esta carácea la hemos observado tanto en lagunas litorales con aguas oligohalinas o prácticamente dulces y también en charcas semipermanentes continentales. La temperatura

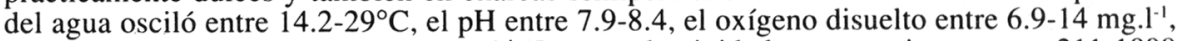
y la alcalinidad entre $1.79-2.72$ meq. $1^{-1}$, Las conductividades se movieron entre 211-1998 $\mu \mathrm{S} . \mathrm{cm}^{-1}$, valores propios de aguas dulces. Por lo que se refiere a la concentración de cloruros y sulfatos, estos oscilaron entre 1-192 mg. $\mathrm{l}^{-1}$ y 3.2-1260 mg. $\mathrm{l}^{-1}$, respectivamente.

En invierno, las poblaciones epifíticas están dominadas por los Clorófitos: Bulbochaete sp. y también masas de Zygnema sp. pl. Además de éstas, también hemos observado diversas bacilariofíceas, principalmente Gomphonema truncatum, aunque también aparecen Achnanthes minutissima y Cocconeis placentula. Durante la primavera, las diatomeas pierden importancia y son substituidas por Aphanocapsa pulchra, Cylindrospermum minutissimum y masas de Oedogonium khannae. En verano se observan muchas especies ticoplanctónicas, como Chroococcus turgidus, C. minutus, Merismopedia punctata, Glaucocystis nostochinearum, Nephrocytium agardhianum o Scenedesmus obliquus, aunque también las hay que son verdaderamente epifíticas, como Achnanthes minutissima, Gomphonema parvulum u Oedogonium khannae.

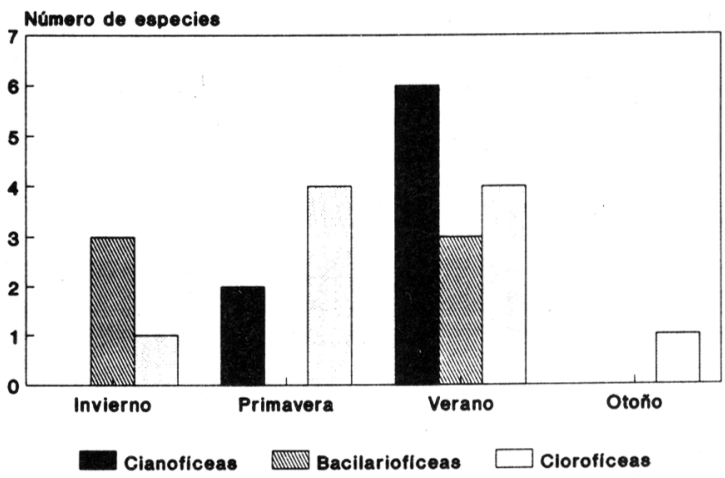

Fig. 2. Variación estacional del número de especies de cianofíceas, bacilariofíceas y clorofíceas epifíticas de Chara hispida. 
Chara polyacantha A. Br. (Fig. 3; Loc.: 5)

Esta carácea solamente fue observada en una localidada (Estanya), en aguas con una alcalinidad muy elevada, que osciló entre 6.08-7.50 meq. $\mathrm{l}^{-1}$, y un $\mathrm{pH}$ relativamente estable, entre 7.2-7.9. Con respecto al oxígeno disuelto se apreció un rango muy amplio de valores, situados entre 2.4-6.6 mg..$^{-1}$. El descenso notable de los valores de oxígeno disuelto se produce en verano, época en que los procesos de descomposición de materia orgánica adquieren importancia en esta laguna de escasa profundidad. Los valores de conductividad fueron moderados $\left(1150-1514 \mu \mathrm{S} . \mathrm{cm}^{-1}\right)$, así como las concentraciones de cloruros $\left(180-218 \mathrm{mg}^{-1}\right)$ y sulfatos (332-458 mg..$\left.^{-1}\right)$.

Las poblaciones que forma Chara polyacantha se mantienen casi todo el año, ya que los talos viejos son periódicamente substituidos por otros jóvenes. Este hidrófito presenta las partes viejas de su talo medianamente incrustadas con carbonato cálcico, y las poblaciones de epífitos perduran durante casi todo el año.

En invierno, predominan de forma mayoritaria las diatomeas como Cocconeis placentula, Cyclotella meneghiniana, Cymbella cesatii, Cymbella cymbiformis, Fragilaria lapponica, Rhopalodia gibba, Synedra ulna y, principalmente, Diploneis ovalis. Esta última especie mantiene una población muy estable todo el año. Durante la primavera, las poblaciones de diatomeas del invierno incrementan ligeramente su abundancia relativa, pero también aparecen otras, como Cyclotella kützingiana y, en menos cantidad, Amphora pediculus y Synedra acus.

Durante el verano y otoño se registra un descenso general del número de especies epifíticas, y Fragilaria lapponica se hace dominante en otoño, acompañada, en proporciones similares por Diploneis ovalis.

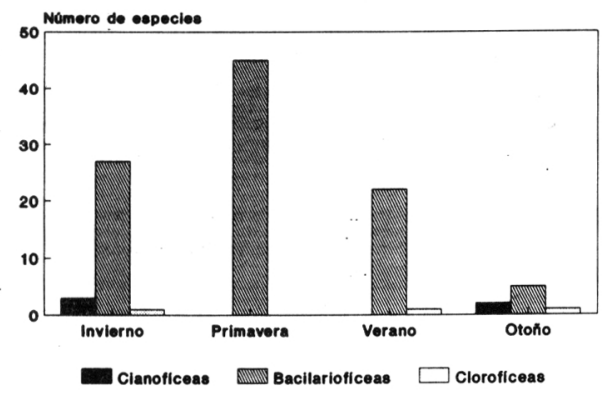

Fig. 3. Variación estacional del número de especies de cianofíceas, bacilariofíceas y clorofíceas epifíticas de Chara polyacantha.

Cladium mariscus (L.) Pohl (Fig. 4; Loc.: 2, 3, 5)

Este helófito fue observado en todos los lagos de tipo cárstico estudiados, en aguas con una alcalinidad desde moderada a notablemente elevada (1.79-7.5 meq. $\left.1^{-1}\right)$, un pH que osciló entre 6.5-8.3 y una variación importante en los valores de oxígeno disuelto (2.4-13.5 mg. $\left.\mathrm{l}^{-1}\right)$. Los valores de conductividad también fueron variables $\left(391-2030 \mu \mathrm{S} . \mathrm{cm}^{-1}\right)$, así como los de los cloruros (9-260 mg..$^{-1}$, ) y los sulfatos (13-1380 mg..$\left.^{-1}\right)$. Estas variaciones se deben a que en Basturs (localidad 3) no se detectan cantidades apreciables de cloruros, ni sulfatos, siendo los bicarbonatos dominantes. Por el contrario, en Banyoles (loc. 2) y Estanya (loc. 5), se observa una importante concentración de sulfatos y, en menor cantidad, cloruros.

Cladium mariscus forma poblaciones durante todo el año, sus tallos viejos suelen presentar más epífitos que los jóvenes y, en general, se observa una predominancia de las bacilariofíceas. Durante la primavera observamos importantes poblaciones de Amphora pediculus y Cymbella cymbiformis, acompañadas por Achnanthes minutissima, Amphora lybica, Cyclotella kützingiana, Cymbella cesatii,Diploneis ovalis y Gomphonema angustum, en menor cantidad. 
En verano, Amphora pediculus pierde importancia, y el epifiton pasa a estar dominado por Cyclotella kützingiana, Fragilaria lapponica y Gomphonema angustum. Acompañando estas especies, pero en cantidades inferiores encontramos cianofíceas, como Chroococcus minutus y C. turgidus, la clorofícea Gloeoplax weberi.

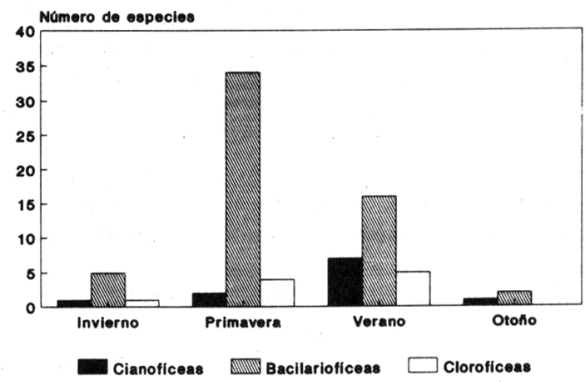

Fig. 4. Variación estacional del número de especies de cianofíceas, bacilariofíceas y clorofíceas epifíticas de Cladium mariscus.

Fontinalis hypnoides Hartm. (Fig. 5; Loc.: 4)

Este musgo acuático forma densas poblaciones en riachuelos del cabo de Creus, en aguas cuya temperatura osciló entre $8-16^{\circ} \mathrm{C}$, un $\mathrm{pH}$ entre 6.6-7.9, una conductividad de 323$1230 \mu \mathrm{S} . \mathrm{cm}^{-1}$ y una alcalinidad de $0.57-2.36$ meq.1 $1^{-1}$. Fontinalis establece poblaciones más o menos importantes durante todas las estaciones del año, a excepción de verano, época en que suele desaparecer. Muestra un desarrollo máximo durante la primavera, época en la que también observamos una mayor abundancia de las especies epifíticas.

En invierno, Fontinalis aparece recubierto por Aphanochaete repens, Epithemia turgida, Gomphonema truncatum, Melosira varians y Rhopalodia gibba, en cantidades muy similares. Estas especies incrementan sus poblaciones durante la primavera, excepto Aphanochaete, ausencia que podríamos atribuir al intenso crecimiento de los talos de Fontinalis, que solo pueden ser colonizados por especies con una tasa de reproducción elevada, como algunas bacilariofíceas. Esta posibilidad la puede confirmar en principio la aparición de especies como Cocconeis placentula o Navicula radiosa.

En verano, los charcos donde crece Fontinalis quedan secos, desapareciendo a su vez los epífitos. En otoño, se vuelven a desarrollar los talos de Fontinalis, que son colonizados por Epithemia turgida, Gomphonema truncatum, Rhopalodia gibba y Synedra acus.

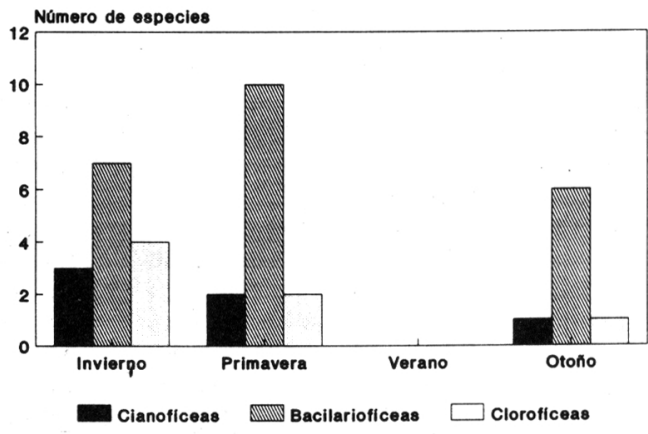

Fig. 5. Variación estacional del número de especies de cianofíceas, bacilariofíceas y clorofíceas epifíticas de Fontinalis hypnoides. 
Myriophyllum spicatum L. (Fig. 6; Loc.: 2, 3)

Este macrófito se ha observado exclusivamente en lagos de tipo cárstico, en aguas cuya alcalinidad se movió entre 3.23-5.22 meq. $\mathrm{l}^{-1}$, la conductividad entre 391-1102 $\mu \mathrm{S} . \mathrm{cm}^{-1}$, los cloruros entre $9-36 \mathrm{mg} . \mathrm{l}^{-1}$ y los sulfatos entre 9-655 $\mathrm{mg} . \mathrm{l}^{-1}$.

En invierno observamos pocos epífitos, tan sólo algunas especies ticoplanctónicas, como Crucigenia tetrapedia, Golenkinia brevispina y Monoraphidium contortum. Durante la primavera, tampoco aparecen especies verdaderamente epifíticas, estando más representadas las ticoplanctónicas, como Coelastrum astroideum, Pediastrum boryanum, $P$. integrum y Sphaerocystis schroeteri. En verano, en cambio, observamos la presencia de Oedogonium crispum y Protoderma viride, ocupando las partes viejas, pero bien iluminadas, de los tallos. En otoño, sobre las partes jóvenes, crecen Microthamnion strictissimum y especies ticoplanctónicas, como Monoraphidium contortum, Scenedesmus ovalternus y S. sempervirens.

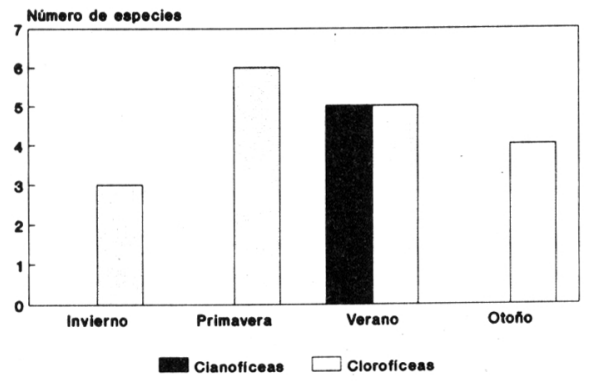

Fig. 6. Variación estacional del número de especies de cianofíceas, bacilariofíceas y clorofíceas epifíticas de Myriophyllum spicatum

Ruppia maritima L. (Fig. 7; Loc.: 1, 7)

Especie propia de lagunas litorales y sistemas lacustres continentales de tipo endorreico. Las aguas en la que fue recolectada presentaron una notable variación anual en su físicoquímica, ya que en la laguna de la Tancada (loc. 7) se observan períodos en los que el agua es prácticamente dulce (primavera-verano) y otras épocas en las que el agua es prácticamente marina. Por el contrario en la laguna de Altafulla (loc. 1), se presenta una situación inversa, es decir, con un período de aguas dulces desde otoño a la primavera y un período mesohalino en verano. Ello explica las notables variaciones que hemos registrado en los valores de la conductividad (4250-19680 $\left.\mu \mathrm{S} . \mathrm{cm}^{-1}\right)$, de los cloruros (1860-19600 mg. $\left.\mathrm{l}^{-1}\right)$ y de los sulfatos (500-2200 mg..$\left.^{-1}\right)$. La temperatura del agua osciló entre $16.5-31.6^{\circ} \mathrm{C}$, el pH entre $7.6-9.4$, el oxígeno disuelto entre 5.4-14.4 mg. $\mathrm{l}^{-1}$ y la alcalinidad entre $1.71-5.96$ meq..$^{-1}$.

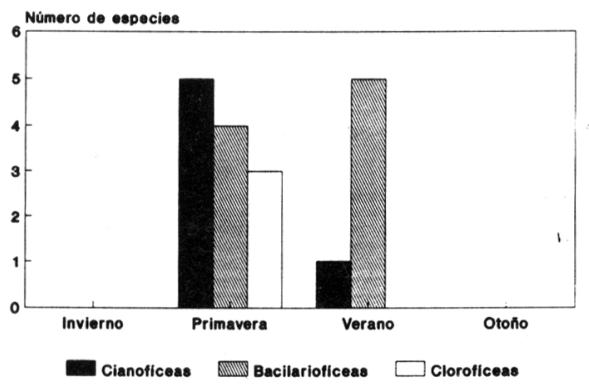

Fig. 7. Variación estacional del número de especies de cianofíceas, bacilariofíceas y clorofíceas epifíticas de Ruppia maritima. 
Durante la mayor parte del año, las plantas de Ruppia se mantienen libres de epífitos, pero cuando las condiciones del medio son diferentes, es decir, cuando las lagunas litorales reciben aguas dulces (abril-mayo), se produce una degradación de las plantas de Ruppia o, por lo menos de su epidermis. En estas situaciones aparece una flora epifítica, principalmente constituida por Calothrix clavata, Cocconeis pediculus, $C$. placentula, Lyngbya epiphytica, Lyngbya kützingii, Melosira juergensii y Rhoicosphenia abbreviata.

\section{Typha angustifolia L. (Fig. 8; Loc.: 2, 5, 6)}

Se trata de un helófito que mantiene su tallo submergido durante la mayor parte del año. Se ha observado principalmente en lagos cársticos y en una laguna semipermanente, en general en aguas con una importante reserva alcalina, así los valores de alcalinidad fueron relativamente elevados $\left(1.79-7.5^{-1}\right)$, el $\mathrm{pH}$ fue variable (6.9-8.3), así como la temperatura $\left(6.4-29^{\circ} \mathrm{C}\right)$, el oxígeno disuelto (2.4-10.4 mg.l $\left.\mathrm{l}^{-1}\right)$, la conductividad $\left(719-2030 \mu \mathrm{S} . \mathrm{cm}^{-1}\right)$, los cloruros $(12-260$ mg..$\left.^{-1}\right)$ y los sulfatos $\left(190-1380 \mathrm{mg} \cdot \mathrm{l}^{-1}\right)$.

El epifiton de Typha angustifolia está dominado por las bacilariofíceas, pero también son importantes las clorofíceas. En invierno observamos desarrollos de Chroococcus turgidus, Cocconeis placentula, Epithemia turgida, Melosira varians, Navicula radiosa y Rhopalodia gibba. Esta última especie forma importantes desarrollos durante la primavera, época en que puede llegar a hacerse dominante. Así mismo, bien entrada la primavera, observamos un importante contingente de especies pertenecientes a diversos grupos algales, entre éstas destacan Aphanochaete repens, Achnanthes minutissima, Diploneis ovalis, Epithemia turgida, Gomphonema truncatum, Melosira varians, Synedra acus, entre otras y, sobre todo, Rhopalodia gibba.

En verano, como en otras plantas acuáticas, se produce una disminución del número de especies epifíticas, apareciendo solamente Characium ensiforme.

Durante el otoño, los tallos jovenes presentan muy pocos epífitos, y solamente hemos observado especies ticoplanctónicas como Gloeocystis vesiculosa, Scenedesmus acutus y $S$. obliquus, que aparecen ocasionalmente entre las poblaciones epifíticas.

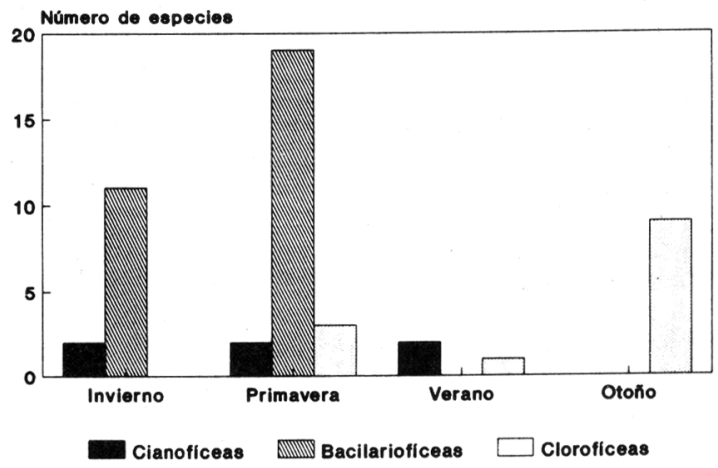

Fig. 8. Variación estacional del número de especies de cianofíceas, bacilariofíceas y clorofíceas epifíticas de Typha angustifolia.

\section{DISCUSIÓN}

Los cambios estacionales observados en poblaciones de algas epifíticas obedecen a diversos factores. Por un lado, las variaciones de luz y temperatura a lo largo del año juegan un papel importante en el crecimiento de numerosas especies (Graham et al., 1982; Whitton, 1970). Asímismo, las variaciones dentro de una estación del año son 
más importantes que las que se producen dentro de un mismo mes (Jones \& Mayer, 1983). A este nivel, adquiere importancia la disponibilidad de nutrientes y la densidad de crecimiento de los macrófitos, entre otros factores.

La predominancia del grupo de las bacilariofíceas en las poblaciones epifíticas observada coincide con la señalada por diversos autores (Jones \& Mayer, 1983; Kairesalo, 1984; Bowker \& Denny, 1980). En los macrófitos estudiados resalta Chara polyacantha, recubierta casi exclusivamente por diatomeas. Por otro lado, se ha señalado que en verano Achnanthes minutissima es la especie dominante entre las poblaciones epifíticas (Klarer \& Hickman, 1975; Jones \& Meyer, 1983; Cattaneo \& Kalff, 1979; Kairesalo, 1984), hecho que coincide en parte con nuestros datos (p.ej. Ceratophyllum demersum), aunque esta diatomea también la hemos observado en abundancia en invierno sobre Chara hispida y en primavera sobre Cladium mariscus.

Por otra parte, a finales de verano u otoño algunos autores (Jones \& Meyer, 1983) destacan la presencia de cianofíceas, ya sea provenientes del plancton como bentónicas. Éste extremo también ha sido corroborado en algunos de los macrófitos estudiados. En efecto, Lyngbya epiphytica aparece en abundancia a finales de verano sobre Ceratophyllum demersum, Chroococcus turgidus y Merismopedia punctata lo hacen sobre Chara hispida y Chroococcus turgidus y C. minutus sobre Cladium mariscus.

Con respecto al estado en que se encuentra el macrófito, cabe señalar que Kairesalo (1984) señala la presencia de numerosos epífitos y un máximo de la biomasa sobre plantas acuáticas en las primeras fases de descomposición, hecho que también hemos observado en Chara hispida y, sobre todo, en Ruppia maritima. El mismo autor señala que cuando el crecimiento de los macrófitos es intenso y estos forman poblaciones muy densas, los valores de la biomasa epifítica descienden, además, añade que en esta situación existe una notable acción de los invertebrados herbívoros, los cuales ingieren gran cantidad de especies epifíticas. Todo ello determina una recesión de las poblaciones de algas epifíticas. En los macrófitos estudiados se aprecia un descenso de la riqueza florística en verano en casi todas las especies estudiadas, extremo que se podría relacionar con los factores considerados por Kairesalo (1984). Cabe destacar en este sentido, el notable descenso en número de especies epifíticas observado durante el verano en los tallos de Typha angustifolia.

AGRADECIMIENTOS. Expreso mi agradecimiento a Assumpció Domínguez, Maria Egidos, Núria Ivorra, Marta López, Tomàs Santalucía y M. Dolores Sierra su ayuda en las campañas de recolección.

\section{BIBLIOGRAFÍA}

BOWKER, D.W. \& P. DENNY -1980- The seasonal succession and distribution of epiphytic algae in the phyllosphere of Lemna minor L. Arch. Hydrobiol.,90(1): 39-55.

BROWN, S.D. \& A.P. AUSTIN -1973- Spatial and temporal variation in periphyton and physicochemical conditions in the littoral of a lake. Arch. Hydrobiol., 71: 183-232.

CAMBRA, J. -1987- Observaciones sobre el perifiton de macrófitos en sistemas lacustres del N.E. de España. Act. IV Congr. Esp. Limnología: 105-114.

CAMBRA, J. - 1991 a-Contribució a l'estudi de les algues epifítiques dels estanys de Banyoles, Basturs i Estanya. Orsis, 6: 27-44.

CAMBRA, J. -1991b- Distribución de las algas epifíticas en sistemas lacustres de Cataluña (N.E. de España). Bot. Complutensis. (en prensa).

CAMBRA, J. -1991c- Contribució al coneixement de les algues epifítiques en llacunes litorals. Collect. Bot. (en prensa). 
CATTANEO, A. J. KALFF -1979- Primary production of algae growing on natural and artificial macrophytes in lake Memphremagog (Que. \& Vt.). Hydrobiologia, 60: 135-144.

GRAHAM, J.M.; AUER, M.T.; CANALE, R.P. \& J.P. HOFFMAN -1982-Ecological studies and mathematical modeling of Cladophora in Lake Huron: 4. Photosynthesis and respiration as functions of light and temperature. J. Great Lakes Res., 8: 100-111.

JONES, R.C. \& K.B. MAYER -1983- Seasonal changes in the taxonomic composition of epiphytic algal communities in lake Wingra, Wisconsin, U.S.A. In: WETZEL, R.G. (Ed.) Periphyton of freshwater ecosystems, 11-16. Dr. Junk Publ. La Haya.

KAIRESALO, T. -1984- The seasonal succession of epiphytic communities within an Equisetum fluviatile L. stand in lake Pääjärvi, southern Finland. Inst. Rev. ges. Hydrobiol., 69(4): 475-505.

KLARER, D.M. \& M. HICKMAN -1975- The effect of thermal effluent upon the standing crop of an epiphytic algal community. Int. Revue ges. Hydrobiol., 60: 17-62.

MOSS, B. -1976- The effects of fertilization and fish community structure and biomass of aquatic macrophytes and epiphytic algal populations: an ecosystem experiment. J. Ecol., 64: 313-342.

STOCKNER, J.G. \& F.A.J. ARMSTRONG -1971- Periphyton of the experimental lakes area, northwestern Ontario. J. Fish. Res. Bd Can., 28: 215-229.

TOMAS, X. -1979- Diatomeas de las aguas epicontinentales de España: géneros Cymbella y Gomphonema. Tesis de Licenciatura. Univ. Barcelona.

WHITTON, B.A. -1970- Biology of Cladophora in frehswaters. Water Res., 4: 457-476.

(Aceptado para su publicación en Abril de 1992)

Dirección del autor: Dpto. Biología Vegetal. Unidad de Botánica. Fac. Biología. Univ. Barcelona. Avda. Diagonal, 648. 08028, BARCELONA. ESPAÑA. ' 